Revista LIDER, 37(22), 2020, pp. 25-43

eISSN: 0719-526

ISSN: 0717-0165

\title{
Agentes-ensamblajes socio-técnicos y adopción de tecnologías de transporte eléctrico en empresas de la ciudad de Osorno, Chile
}

\author{
Socio-technical agent-assemblies and adoption of \\ electric transmission technologies in companies of the \\ City of Osorno, Chile
}

\section{Marco Rosas Leutenegger}

Inacap Osorno, Chile.mrosasl@inacap.cl

\section{RESUMEN}

El proceso de adopción de tecnologías de transporte basadas en energía eléctrica es entendido como un complejo entramado de aspectos técnicos, económicos, normativos, financieros y socioculturales. El objetivo de este trabajo fue identificar agentes, intereses, enrolamientos y dispositivos socio-técnicos específicos que se despliegan en el marco del proceso de adopción de tecnologías de transporte basadas en energía eléctrica, desde una perspectiva sistémica y territorial. Se trató de un estudio descriptivo con enfoque cualitativo, para lo cual fue revisada información secundaria y aplicadas 21 entrevistas semiestructuradas a informantes clave, seleccionados de manera intencional. Se logró constatar la emergencia de al menos 3 dimensiones empíricas, en las que se observa el despliegue de dispositivos, controversias y agenciamientos socio-técnicos: financiero-comercial, políticas público-privadas y marco normativo y científico-tecnológica. Esto llevó a concluir que una perspectiva socio-técnica con enfoque sistémico y territorial, permite hallar nuevos mecanismos de agenciamiento que influyen en el proceso de adopción tecnológica y, además permitió adelantar una propuesta de un modelo socio-técnico de carácter sistémico, con el objeto de desarrollar investigación e intervención a nivel territorial, y de esa manera realizar una contribución al diseño e implementación de políticas públicas e inversiones privadas frente al cambio climático.

Palabras clave: transporte eléctrico; agentes; ensamblajes; adopción de tecnologías

\begin{abstract}
The process of adoption of transport technologies based on electricity is understood as a complex framework of technical, economic, regulatory, financial and socio-cultural aspects. The objective of this paper is to identify agents, interests, enrollments and specific socio-technical devices that are deployed within the framework of the adoption process of transport technologies based on electric energy, from a systemic and territorial perspective. It was a descriptive study with a qualitative approach, for which secondary information was reviewed and 21 semi-structured interviews were applied to intentionally selected key informants. It was possible to verify the emergence of at least 3 empirical dimensions, in which the deployment of devices, controversies and socio-technical arrangements can be observed: financial-commercial, public-private policies and regulatory and scientific-technological framework. This led to the conclusion that a socio-technical perspective with a systemic and territorial approach allows us to find new mechanisms of agency that influence the process of technology adoption and also allowed to advance a proposal for a sociotechnical model of a systemic nature, with the aim to develop research and intervention at the territorial level, and thus make a contribution to the design and implementation of public policies and private investments against climate change.
\end{abstract}

Key words: electric transportation; agents; assemblies; technology adoption

Recibido: 6 de agosto de 2020. Aceptado: 23 de octubre de 2020.

DOI: $10.32735 / S 0719-5265202037179$ 


\section{Introducción}

La adopción de tecnologías de transporte basada en electricidad se vincula con la emergencia de la noción de una "ciudad inteligente", "ciudad digital", "ciudad sustentable" o como se ha sintetizado por su nombre en inglés, Smart City, caracterizada por una fuerte presencia de las tecnologías de información y comunicación (TIC's). Sin embargo, la masificación de tecnologías limpias o sustentables, así como la irrupción de la llamada inteligencia artificial y el uso de datos libres, ha ido ampliando el sentido de lo que entendemos por Smart City. De esta manera, y aunque de modo genérico, la definición de Smart City hace alusión al uso eficiente y racional de recursos en una ciudad por intermedio del desarrollo, uso y aplicación de diferentes técnicas y tecnologías en un área considerada urbana (Walravens, 2012).

Por otra parte, una de las áreas de intervención orientadas a implementar acciones de mitigación contra el cambio climático está relacionada con reemplazar vehículos de combustión interna por vehículos eléctricos, fundamentalmente por su potencial de reducción de emisiones de gases de efecto invernadero ( $\mathrm{CO} 2, \mathrm{NOx}$, etc.) (ONU, 2017). En efecto, y de acuerdo con el Inventario Nacional de Gases de Efecto Invernadero (GEI), el 69\% de las emisiones nacionales en 2013 corresponden al sector de energía, asociadas principalmente al consumo de combustibles fósiles, de lo cual un $28,9 \%$ proviene del sector transporte (Gobierno de Chile, 2017). En el caso de la Región de Los Lagos, esta forma parte de regiones consideradas sumideros netos, vale decir, su balance GEI favorece a su capacidad de absorción y el sector energía corresponde a los sectores emisores de GEI. En tanto, la intensidad de GEI per cápita está levemente por debajo del promedio nacional y la intensidad de GEI por PIB total de la región se encuentra 5 puestos por sobre la media nacional (Gobierno de Chile, 2017). Otra de las externalidades ambientales positivas de las electromovilidades es que esta permite reducir la emisión y carga de residuos, como aceites y lubricantes.

Sin embargo, la aplicación de la electromovilidad, tanto para uso productivo como para el transporte privado o público, requiere analizar y modificar prácticas, equipos y la tecnología existente, lo que supone también considerar inversión pública y privada en infraestructura y capital humano, entre otros aspectos, tal como lo indica la Estrategia Nacional de Electromovilidad, 2017-2022 (Gobierno de Chile, 2017). Si bien existen estudios ligados a los factores y condiciones que hacen posible el desarrollo de la electromovilidad a nivel nacional, no existen suficientes investigacines llevadas a cabo en Chile que describan, comprendan y analicen el proceso de adopción de tecnologías de transporte con energía eléctrica a nivel regional y/o local, y en contextos territoriales alejados de grandes centros urbanos. 
En tal sentido, el proceso de adquisición y adopción de estos medios de transporte, responde a un complejo entramado de ámbitos, que van desde aspectos técnicos, económicos, normativos, financieros hasta socioculturales, que entenderemos para los fines de este trabajo como ensamblajes socio-técnicos. La adopción tecnológica, en términos generales, es concebida como un proceso condicionado por aspectos psico-sociales, desde las perspectivas de usuarios que adoptan equipos y tecnologías. Rogers (1962) identifica perfiles de usuarios en función del momento en que adoptan una tecnología. Los estudios de adopción tecnológica surgen en la década de los '8o (Davis, 1989), notándose un desarrollo teórico por parte de diferentes autores y aproximaciones conceptuales (Venkatesh \& Davis, 2000; King \& He, 2006; Scherer, Siddiq, \& Tondeur, 2019). El concepto ha sido aplicado a diferentes sectores productivos y temáticas, tales como sistemas de producción agropecuarios (Ramírez, Désirée y Velasco, 2013; Rodríguez, Ramírez y Restrepo, 2015), educacional (Scherer, Siddiq, \& Tondeur, 2019). Forero et al (2013) identifican entre las variables explicativas de la adopción de tecnologías agrícolas, aspectos ligados a capital social (capacitación, reciprocidad y organización comunitaria) y capital financiero (planificación financiera). La adopción tecnológica es abordada desde la percepción, las actitudes, la decisión y el comportamiento de usuarios (Koul y Eydgahi, 2018; Sánchez, et. al., 2013). Para la adopción de tecnologías de transporte eléctrico a nivel nacional, los estudios establecen la necesidad de considerar diferentes aspectos tales como: medioambiental, política pública, económico, técnico y social (Almeida, Cardoso y Fuinhas, 2018) y a nivel de usuarios se reconoce la importancia de estudiar aspectos motivacionales en materia medioambiental, financiera y técnica (Peters et al., 2018).

Las formas a través de las cuales se produce el ensamblaje socio-técnico, en este caso con la electromovilidad, es sujeto de los estudios sociales de la ciencia y la tecnología. De este modo, la relación entre desarrollo tecnológico y sociedad, es tratada por Michell Callon. Para ilustrar esta vinculación, Callon planteó, a modo de ejemplo, que los ingenieros desarrolladores del primer auto eléctrico en Francia se movían al campo de análisis sociológico cuando justificaban su elaboración por la existencia y crecimiento de movimientos ambientalistas (Callon, 1993). Se puede afirmar que además de los aspectos estrictamente técnicos, el desarrollo, la transferencia y la difusión tecnológica también se ven influidos por aspectos socioculturales. Siguiendo a Bijker, la teoría del ensamblaje socio-técnico es entendida como un intento para comprender la relación entre tecnología y sociedad (Bijker, 1995). Estos autores llaman la atención acerca de la necesidad de estudiar la relación tecnología-sociedad en la complejidad que la caracteriza y que pareciera estar más allá de lo estrictamente técnico, perfilando una perspectiva sistémica. 
Siguiendo el ejemplo identificado por Urry (2004), el sistema de automovilidad es "entendido como ensamblaje donde se combinan infraestructuras físicas, políticas y sociales, tanto a nivel local como global" (Urry, 2004). De ese modo, las combinaciones horizontales de aspectos multidimensionales serán entendidos como un ensamblaje socio-técnico (Bijker, 1995) que adquieren forma de dispositivos socio-técnicos específicos (Latour, 1995). Para entender el concepto de dispositivo nos remitiremos a la definición de Agamben (2011): "un conjunto de prácticas y mecanismos (invariablemente, discursivos y no discursivos, jurídicos, técnicos y militares) que tienen por objetivo enfrentar una urgencia para obtener un efecto más o menos inmediato", y que empíricamente ha sido tratado para analizar el surgimiento de desarrollos tecnológicos (Padilla y Vásquez, 2018) o, como lo sugiere Ariztía (2016): los dispositivos estructuran nuevas prácticas y ensamblajes.

Estos dispositivos cobrarían características particulares en cada caso y la caracterización de su comportamiento y efectos en el ensamblaje socio-técnico requeriría profundizar en su estudio para facilitar el diseño e implantación de políticas públicas en el sector con visión integral del territorio. A los fines de este estudio intentaremos relevar: ¿cuáles son las características del ensamblaje socio-técnico en la ciudad-territorio de Osorno?, así como, ¿cuáles son los dispositivos socio-técnicos específicos que estarían caracterizando a la situación en estudio?

El objetivo de este trabajo es identificar agentes, intereses, enrolamientos y dispositivos socio-técnicos específicos que se despliegan en el marco del proceso de adopción de tecnologías de transporte basadas en energía eléctrica en empresas y usuarios de la ciudad de Osorno, Chile.

\section{La ciudad de Osorno: justificación del caso de estudio}

El caso de estudio corresponde a la ciudad de Osorno ${ }^{1}$, capital de la provincia del mismo nombre, situada en la Región de Los Lagos, al sur de Chile. De acuerdo al Censo del año 2017, la comuna de Osorno tiene una población de 172.366 habitantes con una densidad poblacional de casi $170 \mathrm{hbtes} / \mathrm{km}^{2}$, y cuyas actividades económicas más importantes corresponden a los sectores agropecuario, turismo, comercio y servicios. Su crecimiento y despliegue urbano, compuesto por barrios densamente poblados (Francke, Oriente, Rahue, Ovejería, principalmente), ha impactado de alguna manera en un crecimiento del parque automotriz privado, dando origen a proyectos de inversión eimplementación en materia de ciclovías así como la habilitación de puentes e infraestructuras urbanas. Lo anterior ha llevado a las autoridades regionales y locales a idear y diseñar proyectos alternativos que mitiguen problemas

${ }^{1}$ Latitud: -40.5725, Longitud: $-73.135340^{\circ} 34^{\prime} 21^{\prime \prime}$ Sur, $73^{\circ} 8^{\prime} 7^{\prime \prime}$ Oeste 
de congestión vehicular que se presentan en el centro de la ciudad, así como en los espacios de intersección y conexión de los barrios de la ciudad. Cabe destacar que en pleno centro urbano, la ciudad alberga las instalaciones administrativas de una de las empresas más importante en materia de distribución de energía eléctrica del país.

Descrito el espacio urbano de análisis de manera general, el caso de estudio se seleccionó, en primer lugar, por la ausencia de investigaciones que aborden la problemática de la adopción de tecnologías de transporte en la ciudad. Por otra parte, a partir del año 2018 existen diferentes iniciativas, tanto públicas, privadas como académicas, que se orientan a desarrollar la electromovilidad en Osorno y que ameritaron realizar un análisis crítico que permita entender la complejidad del fenómeno. Adicionalmente, se advierte una ausencia de enfoques socio-técnicos interdisciplinarios aplicados a la ciudad de Osorno, que haya combinado tanto conocimientos y experiencias provenientes de las ingenierías (mecánicas, eléctrica, entre otras) como de las ciencias sociales. Por último, este artículo constituye uno de los resultados comprometidos en el proyecto Desarrollo de un Modelo de Inteligencia Artificial para la Optimización del Rendimiento de Baterías Ion-Litio en Vehículos Eléctricos, financiado por la Agencia Nacional de Investigación y Desarrollo (ANID) de Chile, y que se implementa en la ciudad de Osorno.

\section{Materiales y métodos}

Este artículo siguió un diseño investigativo de tipo descriptivo-exploratorio de carácter cualitativo. Fue seleccionada esta metodología porque permite estudiar situaciones particulares, profundizar sobre fenómenos acotados o estudiar hechos y percepciones poco conocidos, como es el caso de estudio que se presenta en este artículo (Canales, 2006). Por otra parte, La ventaja de esta metodología es que permite mayor flexibilidad al momento de la recolección de datos e información, aumentando las probabilidades para comprender categorías emergentes del fenómeno.

La metodología aplicada para esta investigación fue la etnografía. La etnografía se ha aplicado, por ejemplo, a estudios urbanos y problemas ambientales (Escuela de Chicago durante principios del S. XX), y generalmente suele implementarse en contextos locales específicos, pues permite profundizar sobre una realidad en un tiempo y espacio dados, mas no generalizar sus hallazgos para otros contextos. Por su parte, los estudios socio-técnicos que han investigado acerca de conceptos como dispositivos y/o ensamblajes socio-técnicos, han implementado metodologías etnográficas para fenómenos urbano-territoriales, como es sugerido por Estévez (2016), notándose la aplicación de otras técnicas como observaciones en terreno y observación participante, lo que permite enriquecer el conocimiento emergente. 
Dado lo anterior, esta investigación trianguló 3 tipos de técnicas, todas las cuales fueron aplicadas durante el año 2019. La primera fue la revisión de documentación e información secundaria, tales como notas de prensa en diarios nacionales y locales, documentos de instituciones y entidades públicas e internacionales, así como instrumentos de planificación territorial (plan regulador y plan de desarrollo comunal de Osorno); La segunda técnica implementada fue la observación participante, a través de la participación del autor en 2 instancias regionales de participación interinstitucional durante el primer trimestre del año 2019, lideradas por la Secretaría Regional del Ministerio de Transporte en la Región de Los Lagos, en las que acudieron diferentes actores regionales, los que para efectos de esta investigación fueron considerados informantes claves, y que permitieron seleccionarlos para una muestra cualitativa; la tercer técnica, correspondió a la entrevista semi-estructurada, las que se aplicaron entre los meses de marzo y agosto del año 2019. Esta técnica se aplicó a diferentes actores que intervienen directamente en el proceso de adopción detecnologías de electromovilidad.

La combinación de técnicas etnográficas, y en particular el uso de la técnica de entrevistas semi-estructuradas, son orientadas a conocer experiencias, prácticas, sentidos y discursos, que permitió ilustrar de mejor manera el funcionamiento y despliegue de ensamblajes de dimensiones socio-técnicas, logrando descifrar y descajanegrizar lo que habría dentro de un proceso de adopción de tecnologías de transporte eléctrico, desde una perspectiva territorialmente situada. Por otra parte, a partir de la muestra descrita en este mismo apartado, permitió conocer procesos emergentes de ensamblajes socio-técnicos a partir de intereses, problemas y soluciones imaginadas, así como evidenciar su combinación con artefactostecnológicos, tal como lo describe Torres para un caso de estudio en Bogotá (Torres, 2012).

Para las entrevistas semi-estructuradas, se trabajó con un muestreo no probabilístico, intencional o, en otras palabras, basado en criterios (Martínez, 2006). Para la elección de los informantes, es decir, aquellos quienes responderán la entrevista, se implementaron diferentes estrategias:

- Técnica de "bola de nieve" o de cadena. Consiste en basarse en los contactos de primeros informantes, hasta saturar la información cualitativa. La fortaleza de esta técnica es que permite actuar en escenarios o situaciones poco conocidas, como es el caso, dada la novedad de la electromovilidad en Osorno. Una de las debilidades de esta técnica de muestreo es que los informantes pueden contar con una reducida red de contactos, así como también es posible que los informantes escogidos transmitan determinada red de conocidos y no de otros.

- Muestreo Intencionado o Teórico, que permitió basarnos en distintos primeros informantes, a partir de lo recogido en la etapa correspondiente a la 
identificación de información secundaria. Esto evitó una las debilidades anteriormente descritas.

- Actores relevantes para la búsqueda deopiniones, vale decir, se escogió a actores que tengan opinión, participación o cercanía respecto a la electromovilidad en Osorno y en particular con el proceso de adopción de tecnologías de transporte eléctrico, o bien, por ser un actor que haya participado en proyectos y/o iniciativas de electromovilidad.

De esta manera, la muestra quedó compuesta por los siguientes actores: a) 3 Ejecutivos comerciales encargados de instrumentos de financiamiento de entidades financieras (bancos con sucursales en Osorno); b) 2 docentes de instituciones de educación superior técnico-profesional que imparten asignaturas en el área académica de mecánica; c) 2 investigadores de Centros de I+D en materia de energía eléctrica y batería; d) 4 miembros de directorios de sindicatos de taxistas en Osorno; e) 3 representantes de empresas privadas, como lo son: comercializadoras de insumos agrícolas, operaciones y flotas de vehículos. Dichos representantes ocupan puestos de dirección de estudios, dirección ejecutiva y/o dirección de inversiones o nuevos negocios; f) 1 representante del Ministerio de Transporte; g) 1 representante del Ministerio de Energía; h) 1 representante del Ministerio del Medio Ambiente; i) 1 representante de la Municipalidad de Osorno, en particular asociado a labores de proyectos urbanos; j) 1 representante de una Empresa generadora y distribuidora de energía; k) 2 representantes de Empresas comercializadoras de vehículos, encargados de líneas de negocio asociado a vehículos eléctricos. La inclusión en la muestra de actores como los sindicatos y gremios de taxi-colectivos es doble: de un lado corresponden a usuarios directamente interesados en adquirir vehículos, modernizar y renovar su flota, así como también son de alguna manera representantes de la sociedad civil en general.

Para el procesamiento y análisis de la información, se siguieron los siguientes pasos: La revisión documental y la información relativa a la intervención en instancias de participación fue recogida en fichas; las entrevistas fueron grabadas y luego transcritas; se realizó una clasificación de la información de acuerdo a las siguientes categorías: financiero-comercial, políticas y marco normativo y soporte y desarrollo científico-tecnológico. Se realizó un proceso de triangulación entre los resultados, los referentes teóricos considerados pertinentes al caso, y la propia pre-comprensión del fenómeno por parte del investigador.

Bajo la noción de dispositivo socio-técnico se buscó los siguientes elementos que lo constituirían: a) prácticas desplegadas por agentes para enrolar estrategias que faciliten/obstaculicen la adopción de vehículos eléctricos; b) mecanismo discursivo, tales como documentos, protocolos, relatos que faciliten/obstaculicen la 
adopción tecnológica; c) mecanismo técnico o artefacto tecnológico, que sea concebido por algún agente como facilitador/obstaculizador de vehículos eléctricos.

\section{Análisis y discusión de hallazgos empíricos}

La investigación permitió constatar la emergencia de, al menos, 3 dimensiones empíricas, en las que podemos observar el despliegue de dispositivos, controversias y agenciamientos socio-técnicos. A continuación se examinan cada una de las dimensiones encontradas, y cuando corresponda, las citas de las entrevistas realizadas a los agentes que permitieron ilustrar cada uno de los conceptos.

\subsection{Financiero-comercial}

Esta categoría permitió analizar aspectos específicos ligados con estrategias financiero-comerciales de entidades financieras o empresas proveedoras de tecnología, tales como sistemas de créditos, mecanismos de posventa, mantención, funcionamiento de ofertas de valor, etc. Asimismo, dentro de esta dimensión pudimos examinar aspectos vinculados con instrumentos públicos de financiamiento, sea subsidios específicos o bien, algún mecanismo depromoción al financiamiento.

Tal como lo indicó un miembro de un sindicato de Osorno, "¿Qué pasa si compramos el vehículo y no tiene mantención ni servicio post-venta?” En este caso, la ausencia del servicio post-venta del vehículo eléctrico funciona como un dispositivo obstaculizador para la adopción tecnológica. De hecho, las ofertas de valor de empresas comercializadoras de automóviles eléctricos dan cuenta del conocimiento que este dispositivo actúa como facilitador: "Nosotros estamos desarrollando los servicios post-venta y capacitando a nuestros funcionarios en China, pero estamos partiendo recién".

En esta materia es interesante constatar la emergencia de tasas preferenciales o especiales para vehículos eléctricos, ya que pueden jugar un rol facilitador. De acuerdo a un representante de una entidad pública, "sería bueno que los bancos dispusieran de tasas preferenciales verdes para la adquisición de vehículos eléctricos". Con ello entendemos que la presencia de tasas verdes especiales se entienden como dispositivos facilitadores. Por su parte, podemos atribuir a esta afirmación un enrolamiento público-privado-usuario, en tanto que el dispositivo es promovido por una entidad pública para que sea implementada por una entidad privada como un banco, para que posteriormente sea utilizada por un usuario final. La presencia de tasas o exenciones tributarias como dispositivos facilitadores, es reconocido por diferentes estudios, en que se plantea que las exenciones de impuestos y subsidios favorecen la adopción de vehículos eléctricos por usuarios (Lévay, Drossinos \& Thiel, 2017). 
Puede encontrarse también que empresas comercializadoras disponen, a modo de dispositivos facilitadores, la agregación de demanda. Tal como lo indica una empresa comercializadora internacional: "puede que el valor del vehículo descienda a medida que podamos juntar unos 20 vehículos". Esto permite indicar que el dispositivo agregación de demanda, enrola al usuario "sindicato de taxistas" y pone en evidencia su capacidad de gestión/movilización de hacer efectiva la demanda. Por su parte los potenciales usuarios manifiestan su disposición a favorecer la agregación de demanda, identificándola como facilitador del proceso de adopción de la tecnología. Su relación y nivel de confianza con el usuario es clave, pues las empresas lo ponen a disposición como un capital social al momento de ofertar su propuesta de valor. Esto coincide con lo planteado por Lazarus, que indica que en los bancos y entidades financieras, en general, se activan agentes y roles asumidos por los empleados bancarios que condicionan (facilitan u obstaculizan) la asignación de financiamiento para la adopción de tecnologías (Lazarus, 2012, en Wilkis, 2014).

De esta manera, ejemplos como los antes mostrados (tasas preferenciales, agregación de demanda, los servicios de post-venta), devienen en mecanismos de agenciamiento, los que, siguiendo a Gaggioli (2014), permiten construir relaciones sociales entre agentes, dotando de significado emocional a su práctica, y permitiendo con ello ensamblar procesos de adopción tecnológica (Gaggioli, 2014). En otras palabras, esto es coherente con lo señalado por Gaggioli (2014 y Knorr-Cetina y Bruegger, 2000), en tanto que los mercados financieros corrresponderían a construcciones socioculturales históricamente dependientes (Gaggioli, 2014), donde actúan traders y dispositivos tecnológicos, como los señalados (Knorr-Cetina y Bruegger, 2000).

Lo anterior permitiría afirmar que el proceso de adopción de tecnologías de transporte eléctrico en la ciudad de Osorno estaría condicionado por un sistema aún inmaduro de financiamiento para la adquisición de vehículos de este tipo, lo que es reconocido en general por los diferentes agentes entrevistados, a pesar del reconocimiento de agentes motivadores tanto aquellos vinculados a entidades financieras como públicas, en particular del Ministerio de Transporte en la Región de Los Lagos. La ausencia de tasas preferenciales que podrían desplegar entidades financieras (tanto público como privadas), finalmente actúa como obstaculizador en Osorno. Lo anterior, sumado a que no existe, desde los gremios de sindicatos de taxis y colectivos, el interés de adquirir vehículos de manera conjunta. Las empresas, por su parte, no ofrecen servicios post-venta a nivel local en Osorno, lo cual va configurando un escenario de ensamblaje socio-técnico adverso para el despliege y desarrollo de la electromovilidad en Osorno. 


\subsection{Políticas y marco normativo}

Correspondió en esta categoría analizar los aspectos ligados al diseño e implementación de estrategias o políticas públicas, aspectos regulatorios como sistemas tarifarios, sistemas de homologación de tecnologías o equipos, articulación, duplicidad, o grado de ajuste con instrumentos de planificación regional o local, etc.

Respecto a esto, el Ministerio de Energía de Chile dispuso el año 2018 una Estrategia de Electromovilidad. Esto permitió que a nivel regional se desplegaran mesas sobre electromovilidad, las que, entre otros aspectos, permitieran articular agentes y relevar la temática. La estrategia movilizó a agentes hacia la toma de decisiones de adoptar tecnologías, tal como se concluye en estudios en la Unión Europea (Cansino, Sánchez-Braza y Sanz-Díaz, 2018; Almeida, Cardoso y Fuinhas, 2018), que indican qué políticas específicas pueden acelerar el proceso de adopción de tecnologías de transporte eléctrico.

Esta estrategia, además, permitió la adhesión de instituciones de educación superior, con actividades específicas como adecuaciones de planes de estudio, implementación de laboratorio o compra de vehículos, acciones que serán analizadas más adelante. En ese sentido, la estrategia actuó como dispositivo facilitador, permitiendo enrolar diferentes agentes.

Por su parte, en esta misma dimensión, puede destacarse lo señalado por el representante de un banco: "nosotros no tenemos instrumentos o políticas específicas de electromovilidad, aunque sí tenemos créditos especiales a eficiencia energética o energía renovable", lo que permite indicar que la presencia de una política específica de un banco aparece como facilitador del proceso de adopción tecnológica.

Por otra parte, en relación a las estrategias y políticas desplegadas, es interesante constatar la emergencia de controversias socio-técnicas latentes. En ese sentido, puede observarse un desajuste entre las prioridades institucionales del Ministerio de Ambiente y el municipio, por un lado, y los ministerios de Transporte y Energía. En efecto, la electromovilidad no constituye necesariamente un ámbito de acción prioritario para resolver los principales problemas socio-ambientales de Osorno, que están asociados al consumo de leña. El representante regional del Ministerio del Medio Ambiente indica: "para nosotros los esfuerzos en materia ambiental en Osorno tienen que ver con la reducción de la contaminación atmosférica producto de la calefacción, y si bien puede ser interesante la electromovilidad, aún no lo tenemos como una prioridad para Osorno". Esto contrastaría con lo sugerido en estudios en los que se releva que la variable ambiental se encontraría entre las razones más importantes para adoptar tecnologías de transporte eléctrico (Peters et al., 2018). Lo interesante de este hallazgo es evidenciar la contradicción entre políticas nacionales con las prioridades a nivel territorial en 
materia de transporte. Puede afirmarse que, si bien en la Región Metropolitana la electromovilidad aparece como una prioridad para efectos de reducir las emisiones de gases de efecto invernadero, esta realidad no necesariamente es coincidente con ciudades de menor densidad poblacional. En ese sentido, las políticas de electromovilidad en ciudades de densidad poblacional como Osorno, aparecen como un proceso de transformación y modernización tecnológica, pero no necesariamente como parte de una política o aspecto medioambiental.

En esta misma línea, puede ilustrarse esta controversia latente, en lo señalado por la Editorial de El Diario Austral de la ciudad de Osorno, de fecha 29 de enero de 2019 que titula: "Buses eléctricos y el Plan pendiente", exponiendo entre otras cosas que: “...si bien este anuncio no viene a solucionar la larga espera del plan de ordenamiento del transporte terrestre de la capital provincial ni la contaminación atmosférica (la que se debe, hay que recordarlo, al uso incorrecto de la leña y no a la emisión de los motores a combustión), el programa de electromovilidad osornino servirá, primero, para incorporar un recorrido muy necesitado que hoy no existe en la ciudad. Y segundo, para ayudar a masificar, desde la toma de conciencia en la ciudadanía, la incorporación de una nueva variable energética en el transporte". (Diario El Austral de Ososrno pág. 8, 29/01/2019). Esto da cuenta que medios de comunicación problematizan el alcance de la electromovilidad respecto a su impacto medioambiental y, por lo tanto, podría perder legitimidad. Sin embargo, es interesante poder discutir estas aseveraciones con nuevas soluciones tecnológicas que puedan implementarse, como la posibilidad que los mismos cargadores eléctricos sirvan para la calefacción de redes internas a nivel domiciliario.

Por ejemplo, la existencia de subsidios y su funcionamiento. Tal como indicó el representante del Ministerio de Transporte: "nosotros como ministerio tenemos el instrumento Renueva tu colectivo y daremos prioridad a los que postulen con vehículos eléctricos". Y añade, "pusimos criterios de evaluación específicos que fijen prioridad en autos eléctricos, sin embargo, la decisión de postular es de los propios dueños o usuarios de las flotas o los vehículos". Ambas medidas aparecerían en teoría como dispositivos facilitadores. Sin embargo, su presencia no es suficiente. Es interesante que esto permitió generar condiciones para la conformación de la mesa de electromovilidad de la Región de Los Lagos. Este hallazgo es complementario con lo descrito en estudios sobre política de electromovilidad, en que se sugiere que cada país disponga de mecanismos de financiamiento específicos acorde a su nivel de desarrollo por (Xingping, et al., 2014).

Un representante de una empresa comercializadora de vehículos indica "que es importante considerar qué tipo de homologación del vehículo y cargadores, si es la China o la francesa, porque puede existir un vacío legal ahí". La puesta en marcha de sistemas de homologación constituye un dispositivo facilitador para los diferentes 
agentes del entramado socio-técnico. Sin embargo, la falta de claridad de la que adolece todavía la política pública, por la incertidumbre o falta de seguridad jurídica, podría estar obstaculizando el proceso en alguna medida. Asimismo, el marco normativo no está ajustado adecuadamente para algunas iniciativas puntuales. Por ejemplo, para proyectos de reconversión de vehículos eléctricos, el representante de una empresa de flotas de vehículos, indica: "Nosotros vamos a apoyar el proyecto, pero no sabemos que luego de reconvertido, podemos usarlo pues el permiso de circulación está asociado a una patente de vehículo de combustión”. En ese sentido, la ausencia de permisos de circulación pone de manifiesto que se requieren tramitaciones especiales para que empresas o usuarios adopten medidas de transformación de vehículos a combustión.

Estos hallazgos son coincidentes con estudios que establecen que tanto la disposición de cargadores, sistemas de homologación y permisos de circulación permiten acelerar procesos de adopción de vehículos de transporte eléctrico (Ryghaug yToftaker, 2016; Karavalakis, et al., 2009 citado en Sampietro, et al. 2019).

Por otra parte, un informante de una institución técnico-profesional, "hoy, por ejemplo, no tenemos una normativa para la manipulación de baterías de vehículos ni tampoco protocolos asociados". Esto es particularmente serio por los riesgos asociados a la manipulación de elementos de estas características. Si bien en Europa y en países desarrollados este tipo de protocolos ya están operativos, en América Latina aún no se han desarrollado. Esto ha sido también relevado en informes sobre la introducción de la electromovilidad en Latinoamérica, en los que se reconoce una brecha en materia de manejo de baterías (Ardanuy Ingeniería, 2019). Los vacíos legales, todavía no resueltos, parecen comportarse como serios obstaculizadores del proceso.

\subsection{Científico-tecnológica}

La última dimensión refiere al ámbito científico y tecnológico, es decir, cómo desarrollos tecnológicos de universidades, centros o entidades de educación superior, así como planes de estudio y el funcionamiento de tecnologías específicas, pueden ser entendidos como dispositivos facilitadores u obstaculizadores.

Asimismo, esta categoría refiere a la infraestructura tecnológica habilitante, provista por empresas proveedoras de tecnologías, así como nuevo conocimiento, nuevas tecnologías o tecnologías emergentes desarrolladas por entidades como centros científico-tecnológico o instituciones de educación superior.

Para ilustrar los dispositivos referidos a esta dimensión y su funcionamiento específico, podemos mencionar lo indicado por un entrevistado del sindicato de taxis y colectivos, quien manifiesta su preocupación cuando afirma: "Si. por ejemplo, todos adquirimos vehículos eléctricos, y hay 2 cargadores, tendremos que hacer 
fila para cargar nuestros vehículos, entonces tenemos que saber si además del vehículo, tenemos que comprar cargadores domiciliarios". De esta manera, no solamente la presencia del cargador corresponde a un dispositivo facilitador, sino también la cantidad y su ubicación, la que además depende del uso, el costo y el flujo de carga. Tal como se comentó, es consistente con estudios en los que se relevan aspectos relacionados con infraestructura tecnológica (Ryghaug y Toftaker, 2016; Karavalakis, et al., 2009 citado en Sampietro, et al. 2019).

En este ámbito también puede mencionarse la batería del vehículo como dispositivo socio-técnico, y su uso, ciclos de carga, cadencia, vida útil, etc. corresponden también a obstaculizadores que hacen que pudiera haber mayor o menor resistencia a la adopción tecnológica. Lo anterior es consistente con lo señalado por una empresa distribuidora de energía, cuando un representante señala que "Nos comprometemos con dejar instalados dos cargadores de vehículo eléctricos". Este dispositivo es trabajado por Dellavalle y Zubaryeva (2019), en que es destacada la relevancia de la batería y su estado de salud.

Uno de los dispositivos que puede mencionarse, es lo que indica el representante del Ministerio de Energía: "estamos trabajando con la U. de Chile en el diseño de una aplicación informática para el rastreo de movimiento de flotas y optimización de baterías”. Esta afirmación permite identificar dos aspectos: por un lado, la aplicación deviene en dispositivo que actúa como agenciamiento entre dos agentes; y por otra parte, es reconocido como un dispositivo que facilita el proceso de adopción tecnológica. Aplicaciones informáticas, y en general innovaciones relacionadas con electromovilidad, han identificado factores tecnológicos como variables independientes al proceso de adopción tecnológica (Almeida, Cardoso y Fuinhas, 2018).

En este ámbito, por ejemplo, el representante del Ministerio de Transporte señala que "nos interesa pronto que se pueda sacar el primer técnico en mantención de vehículos eléctricos o que abran un programa de capacitación sobre motores eléctricos, porque eso los taxistas y colectiveros no lo saben". Los programas de formación curricular actúan como dispositivos socio-técnicos facilitadores, así como también actividades de extensión o capacitación específicas, y por eso es que un representante de una institución de educación técnica indica: "estamos viendo si cambiamos el programa de estudio, o simplemente adecuamos algunos ramos", esto que permite contrastarlo con lo estudiado por Larsson y Holmberg (2018), que relevan la función del sistema de educación superior, como un laboratorio de aprendizaje interactivo.

Lo mismo puede señalarse respecto a la implementación de talleres, laboratorios y equipamiento tecnológico en materia de electromovilidad, tal como lo señala un académico de una institución de educación técnico-profesional: "Cuando 
habilitemos el laboratorio de electromovilidad, podemos hacer reconversiones y pruebas para nuevos vehículos, así como prestar servicios específicos”. El equipamiento y el laboratorio sirven no solamente para hacer pruebas o investigaciones, sino que para aumentar la certidumbre técnica asociada a la formación de servicios de mantención.

Como es posible observar, Osorno es actualmente una ciudad donde están en etapas de diseño e implementación diferentes proyectos científico-tecnológico en materia de electromovilidad: instalación de cargadores eléctricos, habilitación de taller de electromovilidad en institución de educación superior técnico-profesional, corredor de buses eléctricos con instalación de cargadores, proyectos de desarrollo tecnológico, los que van configurando un escenario dinámico de enrolamientos socio-técnicos de intereses orientados a adoptar tecnologías de transporte eléctrico. Sin embargo, tal como se aprecia, aún son proyectos e iniciativas que están en una fase incipiente de desarrollo. En esa línea, sería interesante avanzar en el seguimiento y monitoreo et nográficamente de su evolución para trazar dinámicas de sentidos.

\subsection{Balance y perspectivas}

La investigación permitió afirmar que la adopción de tecnologías de transporte basadas en energía eléctrica se da en el marco de una red de relaciones complejas, socio-técnica, dinámicas y sistémicas, de multinivel y multi-agente. Para el entendimiento de este fenómeno, no bastaría, de esta manera, con una mirada puramente cuantitativa basada en los recursos y/o equipamiento de infraestructuras urbanas, o bien, de enfoques funcionalistas desarrollados en estudios urbanos. Se haría necesario desarrollar estudios socio-técnicos que permitan entender que la adopción de tecnologías eléctricas se da en condiciones de enrolamientos de intereses, construcciones de sentido y percepciones, así como en instancias complejas de interacción de culturas y prácticas. Una perspectiva socio-técnica permite hallar nuevos procesos específicos de ensamblajes. Es decir, mecanismos de agenciamientoyenrolamiento que están en juego en el proceso de adopción tecnológica.

Esto complementa un enfoque psico-social centrado en individuos. Nos hacemos cargo de componentes de validación y legitimación específicos, recogiendo el fenómeno de apropiación, que podría facilitar en definitiva la sostenibilidad en el tiempo de la adopción de la tecnología. En otras palabras, si bien los estudios psicosociales han mostrado un interesante abordaje para relacionar variables individuales y perceptuales, los resultados de este artículo permitirían abrir nuevas líneas argumentativas que entiendan el proceso de adopción desde una perspectiva dinámica que no actuaría sino de manera reticular, dotada además de significados y sentidos. 
En términos metodológicos, de las limitaciones de este estudio fue la no inclusión de movimientos socio-ambientales en la muestra, los que, si bien no están directamente relacionados con el proceso de adopción tecnológica, pudieran, eventualmente, incidir (favorablemente o negativamente) en su agilización/implementación. Dado lo anterior, se recomienda integrar en muestras cualitativas posibles agenciamientos de cara con movimientos socio-ambientales, con lo cual, de forma adicional, podrían emerger seguramente, controversias socio-técnicas en el ámbito de la electromovilidad.

Adicionalmente, comprender este proceso desde una perspectiva sistémicoterritorial permitió evidenciar los (des)ajustes entre estrategias e instrumentos de planificación a nivel nacional e instrumentos de planificación y gestión local, y su vinculación con proyectos de inversión de carácter privado. Esto permite colaborar en el (re)diseño de políticas públicas que afronten directamente esta temática, tales como la agilización de instrumentos y mecanismos de financiamiento para la adquisición de vehículos eléctricos, así como también el diseño de planes reguladores o planes de desarrollo comunal que incluyan estudios y/o información prospectiva de cara a la transformación en el tipo de transporte utilizados en medios urbanos y/o interurbanos. Se haría con ello posible abordar características específicas de territorios donde ocurra el proceso de adopción tecnológica, sin perder la perspectiva sistémica. Si bien podría resultar prematuro extrapolar el caso de Osorno a otras ciudades, sí es posible argumentar que las dinámicas, agentes y dimensiones pueden servir de base para entender el proceso de adopción de la electromovilidad a nivel urbano-territorial, tanto en ciudades similares a Osorno, como otros espacios urbano-territoriales de mayor tamaño y/o densidad poblacional. Es más, podría sostenerse que las dimensiones, agentes, dispositivos, ensamblajes y agenciamientos examinadas en este trabajo, se desplegarían en otras realidades urbanas, de manera indistinta al tamaño y/o densidad poblacional.

El análisis realizado de la información que hemos podido recoger en este estudio y su contrastación con estudios y experiencias anteriores, nos permite presentar una propuesta de un modelo socio-técnico de carácter sistémico y territorial, dado por 3 dimensiones que interactúen entre sí: financiero-comercial, políticas y marco normativo, y soporte y desarrollo científico-tecnológico, en las que se despliegan agenciamientos y controversias, como las que se acaban de identificar; agentes y dispositivos específicos.

Con lo anterior, y tal como se ha indicado, puede avanzarse en construir modelos sistémicos de medición cualitativa y cuantitativa e intervención a nivel territorial público-privada, que impacte en el diseño e implementación de políticas públicas e inversiones privadas, como una política de modernización tecnológica y de cambio climático. 


\section{Bibliografía}

Agamben, G. (agosto de 2011). ¿Qué es un dispositivo? Sociológica (Méx.), 26(73), 249-264. Obtenido de http://www.scielo.org.mx/scielo.php?script=sci_arttext\&pid=So18701732011000200010\&lng=es\&tlng $=$ es.

Almeida, S., Cardoso, A., \& Fuinhas, J. A. (Mayo de 2019). Technological progress and other factors behind the adoption of electric vehicles: Empirical evidence for EU countries. Research in Transportation Economics, 74, 28-39. Obtenido de https://reader.elsevier.com/reader/sd/pii/So739885917303530?token=86B 43D4CEA862284631ED705475DBADo7B9oF19115F201345991A63DC8342 95610823FEEE22F12BBA3827B5B679BCAC6

Antunes da Luz, A., \& Kovaleski, J. L. (2018). Framework Proposal for Management of Knowledge and Technology Transfer in Brazilian Academic Internships. Journal of Technology Management \& Innovation, 13(3), 3-11. Obtenido de https://www.jotmi.org/index.php/GT/article/view/2576

Arciénaga, A., Nielsen, J., Bacarini, A., Martinelli, Silvia, Kofuji, S., \& García, J. F. (Abril de 2018). Technology and innovation management in Higher Education-Cases from Latin America and Europe. Administrative Sciences, 8(2), 1-34. Obtenido de https://www.mdpi.com/2076-3387/8/2/11/htm

Ardanuy Ingeniería. (2019). La electromovilidad en el transporte público. Buenos Aires: CAF. Obtenido de https://scioteca.caf.com/bitstream/handle/123456789/1466/La\%20electro movilidad\%2oen\%2oel\%2otransporte\%2op\%C3\%BAblicode\%2oAm\%C3\%A 9rica\%20Latina.pdf?sequence $=6$

Ariztía, T. (2016). Consumption and the middle classes: three sociological. Polis, 15(43), 435-459. Obtenido de https://dx.doi.org/10.4067/So71865682016000100021

Bijker, W. (1995). Of bicycles, bakelites, and bulbs. Toward a theory of sociotechnical change. Cambridge: The Mit Press.

Callon, M. (1993). Society in the making: The study of technology as a tool for sociological analysis. En W. Bijker, T. Huges, \& T. Pinch (Edits.). Cambridge: MIT Press.

Callon, M., Courtial, J.P, \& Penan, H. (1995). Cienciometría. Gijón, España: Ediciones Trea.

Canales, M. (2006). Metodología de lnvestigación Social. Santiago de Chile: LOM Ediciones.

DellaVAlle, N., \& Zubaryeva. (Septiembre de 2019). Can we hope for a collective shift in electric vehicle adoption? Testing salience and norm-based interventions in 
South Tyrol, Italy. Energy Research \& Social Science, 46-61. Obtenido de https://doi.org/10.1016/j.erss.2019.05.005

Estévez, B. (2016). Controversias, hibridez y diseño urbano: Abrir el candado de la representación y multiplicar los posibles del espacio público. Revista de geografía Norte Grande, pp.7-37.

Gaggioli, N. N. (Febrero de 2020). El mundo financiero como objeto antropológico. Runa, 35(1), 41-60. Obtenido de http://www.scielo.org.ar/scielo.php?script=sci_arttext\&pid=S1851$96282014000100003 \& \ln =$ =es\&tlng=es

Gobierno de Chile. (2017). Estrategia Nacional de Electromovilidad. Santiago. Obtenido de https://www.energia.gob.cl/sites/default/files/estrategia_electromovilidad8dic-web.pdf

Gobierno de Chile. (2018). Ley 21091 sobre Educación Superior. Obtenido de https://www.leychile.cl/Navegar?idNorma=1118991

Karavalakis, G., Alvanou, F., Stournas, S., \& Bakeas, E. (Junio de 2009). Regulated and unregulated emissions of a light duty vehicle operated on diesel/palmbased methyl ester blends over NEDC and a non-legislated driving cycle. Fuell, $88(6), 1078-1085$.

King, W., \& He, J. (2006). A meta-analysis of the technology acceptance model. Information \& Management, 43(6), 740-755.

Koul, S., \& Eydgahi, A. (2018). Utilizing Technology Acceptance Model (TAM) for driverless car technology Adoption. Journal of technology management \& innovation, 13(4), 37-46. Obtenido de https://dx.doi.org/10.4067/So71827242018000400037

Larsson, J., \& Holmberg, J. (Marzo de 2017). earning while creating value for sustainability transitions: The case of Challenge Lab at Chalmers University of Technology. Journal of Cleaner Production , 172, 4411-4420. Obtenido de https://www.sciencedirect.com/science/article/pii/So959652617305073?via $\% 3$ Dihub

Lévay, P. Z., Drossinos, Y., \& Thiel, C. (Junio de 2017). The effect of fiscal incentives on market penetration of electric vehicles: A pairwise comparison of total cost of ownership. Energy Policy, 105, 524-533. Obtenido de https://doi.org/10.1016/j.enpol.2017.02.054

Martínez, C. (2012). El muestreo en investigación cualitativa. Principios básicos y algunas controversias. Ciência \& Saúde Coletiva, pp.613-619.

Padilla, P., \& Vásquez, F. (2018). estión tecnológica e innovación en salud: el caso del HPV Easy Typing para la prevención del Cáncer Cervicouterino. Cultura- 
hombre-sociedad, 26(2), 116-136. Obtenido de https://dx.doi.org/10.7770/o719-2789.2018.cuhso.06.ao6

Peters, A., van del Werff, E., \& Steg, L. (Mayo de 2018). Beyond purchasing: Electric vehicle adoption motivation and consistent sustainable energy behaviour in The Netherlands. Energy Research \& Social Science, 319, 234-247. Obtenido de https://www.sciencedirect.com/science/article/pii/S2214629617303353

Rodríguez, H., Ramirez, C. J., \& Restrepo Betancur, L. F. (2015). Factores que influencian la adopción de tecnología de gestión en producción lechera. Temas agrarios, 20(1), 33-44.

Ryghaug, M., \& Toftaker, M. (Julio de 2016). Creating transitions to electric road transport in Norway: The role of user imaginaries. Energy Research \& Social Science, $17, \quad 119-126 . \quad$ Obtenido de https://www.sciencedirect.com/science/article/pii/S2214629616300925

Samaniego, J., \& Schneider, H. (2017). Financiamiento para el cambio climático en América Latina y el Caribe en 2015. Santiago: Naciones Unidas. Obtenido de https://repositorio.cepal.org/bitstream/handle/11362/41010/1/S1700037_e s.pdf

Sampietro, J. L., Puig, V., \& Castelló, R. C. (2019). Estrategia de gestión de la energía en vehículos eléctricos con pila de combustible y sistema de almacenamiento híbrido utilizando control predictivo económico. Maskay, 9(2), 31-40. Obtenido de https://dx.doi.org/10.24133/maskay.v9i2.1145

Sánchez Toledano, B. I., Zegbe Domínguez, J. A., \& Rumayor Rodríguez, A. F. (2013). Propuesta para evaluar el proceso de adopción de las innovaciones tecnológicas. Revista mexicana de ciencias agrícolas, 4(6), 855-868. Obtenido de http://www.scielo.org.mx/scielo.php?script=sci_arttext\&pid=S200709342013000600003\&lng=es\&tlng=es

Scherera, R., Siddiq, F., \& Tondeur, J. (2019). The technology acceptance model (TAM): A meta-analytic structural equation modeling approach to explaining teachers' adoption of digital technology in education. Computers \& Education, 128, 13-35. Obtenido de https://www.sciencedirect.com/science/article/pii/So360131518302458

Sismondo, S. (2009). An Introduction to science and technology studies. WileyBlackwell.

Torres, A. (2012). Infraestructuras urbanas sociotécnicas. Vender o no vender la ETB - Empresa de Telecomunicaciones de Bogotá. urbe. Revista Brasileira de Gestão Urbana, 263-297. 
Urry, J. (Octubre de 2004). The 'System' of Automobility. Theory, Culture \& Society, 21(4-5), 25-39. Obtenido de https://journals.sagepub.com/doi/10.1177/0263276404046059

Venkatesh, V., \& Davis, F. (2000). A theoretical extension of the technology acceptance model: four longitudinal field studies. Management Science, 46(2), 186-204.

Walravens, N. (2012). Mobile Business and the Smart City: Developing a Business Model Framework to Include Public Design Parameters for Mobile City Services. Journal of theoretical and applied electronic commerce research, 7(3), 121-135. Obtenido de https://dx.doi.org/10.4067/So71818762012000300011

Wiliam, K. H. (2006). A meta-analysis of the technology acceptance model. Information \& Management, 43(6):740-755.

Wilkis, A. (2014). Una sociología moral de las relaciones bancarias. Desacatos, 44, 210-215. Obtenido de http://www.scielo.org.mx/scielo.php?script=sci_arttext\&pid=S1607050X2014000100015\&lng=es\&tlng=es.

Xingping, Z., Jian, X., Rao, R., \& Yanni, L. (214). Policy Incentives for the Adoption of Electric Vehicles. Sustainability, 6, 8056-8078. Obtenido de www.mdpi.com/journal/sustainability 